\title{
Analysis of IgM, IgA, and IgG Isotype Antibodies Directed Against SARS-CoV-2 Spike Glycoprotein and ORF8 in the Course of COVID-19.
}

\section{Denise Meinberger}

Institute for Clinical Chemistry, Medical Faculty, University of Cologne, 50937 Cologne, Germany.

\section{Manuel Koch}

Institute for Dental Research and Oral Musculoskeletal Biology, Institute for Biochemistry II, Center for Molecular Medicine Cologne,Medical Faculty, University of Cologne, 50931 Cologne, Germany.

\section{Annika Roth}

Institute for Clinical Chemistry, Medical Faculty, University of Cologne, 50937 Cologne, Germany.

\section{Gabriele Hermes}

Institute for Clinical Chemistry, Medical Faculty, University of Cologne, 50937 Cologne, Germany.

\section{Jannik Stemler}

Department I of Internal Medicine, Excellence Center for Medical Mycology (ECMM), Chair Translational Research, Cologne Excellence Cluster on Cellular Stress Responses in Aging-Associated Diseases (CECAD), German Centre for Infection Research (DZIF), Partner Site Bonn-Cologne, Medical Faculty, University of Cologne, 50937 Cologne, Germany.

\section{Oliver A. Cornely}

Department I of Internal Medicine, Excellence Center for Medical Mycology (ECMM), Chair Translational Research, Cologne Excellence Cluster on Cellular Stress Responses in Aging-Associated Diseases (CECAD), German Centre for Infection Research (DZIF), Partner Site Bonn-Cologne, Medical Faculty, University of Cologne, 50937 Cologne, Germany.

\section{Thomas Streichert}

Institute for Clinical Chemistry, Medical Faculty, University of Cologne, 50937 Cologne, Germany.

\section{Andreas R. Klatt ( $\square$ andreas.klatt@uk-koeln.de )}

Institute for Clinical Chemistry, Medical Faculty, University of Cologne, 50937 Cologne, Germany.

\section{Research Article}

Keywords: SARS CoV 2, COVID-19, antibody response, immunoglobulin, cross reactivity, spike glycoprotein, ORF8

Posted Date: November 13th, 2020

DOl: https://doi.org/10.21203/rs.3.rs-106292/v2 
License: (c) (i) This work is licensed under a Creative Commons Attribution 4.0 International License. Read Full License

Version of Record: A version of this preprint was published at Scientific Reports on April 26th, 2021. See the published version at https://doi.org/10.1038/s41598-021-88356-8. 


\section{Abstract}

Immunoassays are a standard diagnostic tool assessing immunity in severe acute respiratory syndrome coronavirus type 2 (SARS-CoV-2) infection. However, immunoassays do not provide information about contaminating antigens or cross-reactions and might exhibit inaccurately high sensitivity and low specificity. We aimed to gain insight into the serological immune response of SARS-CoV-2 patients by immunoblot analysis.

We analyzed serum immunoglobulins IgM, -A, and -G directed against SARS-CoV-2 proteins by immunoblot analysis from 12 infected patients. We determined IgG isotype antibodies by commercially available ELISA and assessed the clinical parameters of inflammation status and kidney and liver injury.

We found evidence for antibody cross-reactivity, which calls into question a reliable assessment of serum samples tested negative for anti-SARS-CoV-2 antibodies by immunoassays. Nevertheless, for the detection of IgG anti-SARS-CoV-2 antibodies, our data suggest that the use of the spike glycoprotein in immunoassays should be sufficient to identify positive patients. Using a combination of the spike glycoprotein and the open reading frame 8 protein could prove to be the best for the detection of patients positive for anti-SARS-CoV-2 IgM antibodies. We found that the antibody response alone is not decisive for the course of the disease, but inflammation parameters are promising indicators.

\section{Introduction}

Severe acute respiratory syndrome coronavirus type 2 (SARS-CoV-2) is one of seven known human coronavirus pathogens. Most human coronavirus infections result in the common cold and account for up to $15 \%$ of such cases ${ }^{[1]}$. In contrast, SARS-CoV, MERS-CoV, and SARS-CoV-2 infections can develop into life-threatening lower respiratory syndromes ${ }^{[2-4]}$. As of October 23 , a total of $42,089,893$ infected cases and more than 1,144,274 deaths (mortality 4\%) were reported (WorldOmeter https://www.worldometers.info/coronavirus/). Coronavirus disease 2019 (COVID-19) has resulted in a global pandemic.

Coronaviruses are single-stranded RNA viruses. The SARS-CoV-2 genome encodes few proteins despite its large size of approximately 30,000 bases. These proteins are structural spike, nucleocapsid, membrane, and envelope proteins that are needed to produce structurally complete viral particles ${ }^{[5]}$. Additionally, 16-17 non-structural proteins (ns1 to ns17), such as 3-chymotrypsin-like protease, papain-like protease, helicase, RNA-dependent RNA polymerase ${ }^{[5]}$, and multiple uncharacterized proteins encoded by open reading frame (ORF) 3a, ORF6, ORF7, ORF8, ORF10, are coded for. ORF8 and several proteins from alpha- and beta-coronaviruses define novel families of immunoglobulin (Ig) domains, which might function as potential immune modulators to delay or attenuate the host immune response against the viruses ${ }^{[6]}$. 
Accurate and fast diagnosis of SARS-CoV-2 infection is important to identify COVID-19 patients, limit the extent of the pandemic, and initiate therapeutic measures. Polymerase chain reaction (PCR) is the standard diagnostic tool. Immunoassays are essential to assess the immunity of patients post-infection and to analyze the efficacy of vaccines. Various serological immunoassays have been rapidly developed [7]. The spike glycoprotein of SARS-CoV-2 contains immunodominant epitopes and was used as an antigen in immunoassays to identify antibodies directed against SARS-CoV-2, providing high specificity and sensitivity ${ }^{[8,9]}$. The diagnostic performance of commercially available SARS-CoV-2 immunoassays is particularly good, and the diagnostic sensitivity and specificity are generally higher than $80 \%$ or even $90 \%$. However, immunoassays do not provide information about cross-reactivity or contaminating antigens. This may lead to lower specificity and false high sensitivity. While the number of studies employing immunoassays is rapidly increasing, no study has examined antibody response with more specific methods, namely, immunoblots.

We investigated the serum of 12 COVID-19 patients with mild, moderate, or severe disease for the presence of IgM, -A, and -G isotype antibodies directed towards SARS-CoV-2 spike protein (S), receptor-binding domain (RBD) of SARS-CoV-2 spike protein (R), subunit 1 of the spike protein (S1) and ORF8 protein ( $\mathrm{O}$ ) by immunoblot analysis. Furthermore, we investigated the patient's serum by using a commercially available anti-S1 IgG isotype ELISA. C-reactive protein (CRP), procalcitonin (PCT), and interleukin-6 (IL-6) were determined for the assessment of the systemic inflammatory reaction of the patients.

\section{Materials And Methods Study and ethics}

This case-control study was performed at and under the guidelines of the University Hospital of Cologne and approved by the ethics committee of the Medical Faculty of the University of Cologne (ISI protocol, ID 08-160). Written informed consent was obtained from all patients and controls.

\section{Demographic and clinical characteristics}

The study included 12 patients who tested positive for SARS-CoV-2 infection by PCR. Four showed mild symptoms not requiring oxygen supply (WHO 3), six displayed moderate symptoms requiring non-invasive oxygen supply (WHO 4), and two developed severe symptoms requiring invasive ventilation (WHO 5-7) ${ }^{[10]}$. The latter patients died with underlying chronic disease. The age of the patients with a mild and moderate course of COVID-19 ranged from 31 to 89 years with a median age of 59 years; $50 \%$ were female. The age of the patients with a severe course was between 65 and 88 years, with a median age of 77 years; $50 \%$ were female in this group. The predisposing conditions were hypertension, diabetes, surgery, malignancies, chronic lung disease, and chronic renal diseases. As controls, the serum of three 
donors without SARS-CoV-2 infection was used. The age of these all-male donors ranged from 30 to 50 years with a median age of 38 years.

\section{Blood samples}

Samples were collected between March 27 and Mai 11, 2020, in $4.7 \mathrm{ml}$ serum monovettes (Sarstedt, Germany), centrifuged at $2772 \mathrm{~g}$ for 10 minutes, and stored at $-80^{\circ} \mathrm{C}$.

\section{Cloning and recombinant expression of SARS-CoV-2 proteins}

Spike ectodomain (138.8 kDa) (MN908947; AA: 1-1207), including a C-terminal T4 foldon, RBD (26 kDa) (MN908947; AA: 331-524), and subunit 1 (62.7 kDa) (MN908947; AA: 14-529) regions of the spike DNA as well as ORF8 (16.3 kDa) (MN908947; AA: 16-121), were amplified from synthetic gene plasmids (furin site mutated, K986P and V987P) using specific PCR primers. PCR products were digested with the appropriate restriction enzymes and cloned into modified sleeping beauty transposon expression vectors

${ }^{[11]}$ containing a double Strep II purification tag with thrombin cleavage and a BM40 signal peptide sequence. Expression constructs were transfected into HEK293 EBNA cells using FuGENE® HD (Promega $\mathrm{GmbH}, \mathrm{USA}$ ) in DMEM/F12 (Merck, Germany) supplemented with $6 \%$ fetal bovine serum (Biochrom AG, Germany), generating stable cell lines. After selection with puromycin ( $3 \mu \mathrm{g} / \mathrm{ml}$; Sigma, USA) for four days, cells were induced with doxycycline hyclate $(0.5 \mu \mathrm{g} / \mathrm{ml}$, Sigma, USA). Cell supernatants were harvested every third day and filtered, and the recombinant proteins were purified via Strep-Tactin®XT (IBA Lifescience, Germany) resin. Proteins were then eluted with biotin elution buffer (IBA Lifescience, Germany), dialyzed against Tris- or phosphate-buffered saline (TBS/PBS), and stored at $4^{\circ} \mathrm{C}$.

\section{SDS-PAGE and immunoblot}

Samples were separated on 4-12\% Bis-Tris polyacrylamide gels (Thermo Fisher Scientific, USA) and stained with Coomassie brilliant blue R-250 (Merck, Germany) or using the SilverQuest silver staining kit (Invitrogen Thermo Fisher Scientific, USA). The proteins were transferred to a polyvinylidene fluoride (PVDF, $0.45 \mu \mathrm{m}$ ) membrane (Thermo Fisher Scientific, USA). For the Strep-Tactin®-HRP conjugate (IBA Lifescience, Germany), membranes were blocked in PBS containing 0.05\% Tween 20 and 3\% BSA (PBS-TB) and incubated with Strep-Tactin ${ }^{\circledR}$-HRP conjugate diluted 1:100,000 in PBS containing $0.05 \%$ Tween 20 (PBS-T). Otherwise, membranes were blocked in TBS containing 0.01\% Tween 20 (Merck, Germany), $5 \%$ milk powder (Roth, Germany), and $1 \%$ bovine serum albumin (Sigma, USA) (TBS-TMB) and incubated with the patients' serum diluted 1:200 in TBS-TMB. Finally, the membranes were incubated with cross-adsorbed HRP-conjugated polyclonal goat anti-human $\lg \mathrm{G}(1: 50,000), \lg \mathrm{M}(1: 50,000)$, and $\lg A$ $(1: 20,000)$ (A18841, A18829 Novex Thermo Fisher Scientific, USA; ab98558 Abcam, England) in TBS-TMB. Membranes were treated with Amersham ${ }^{\mathrm{TM}} \mathrm{ECL}^{\mathrm{TM}}$ Prime Western Blotting Detection Reagent 
(Thermo Fisher Scientific, USA), and signals were visualized with the ChemiDoc XRS+ System (BioRad, Germany). Signals were analyzed using Image Lab software version 4.0 from BioRad. Patients were considered positive for anti-SARS-CoV-2 antibodies if their signals exceeded the strongest signal of the controls. Prestained SeeBlue ${ }^{\text {TM }}$ Plus 2 protein standard (Thermo Fisher Scientific, USA) was used for immunoblotting and Coomassie staining.

\section{Enzyme-linked immunosorbent binding assay (ELISA)}

Pre-coated and blocked 96-well plates (IDK® anti-SARS-CoV-2 IgG ELISA, Immundiagnostik AG, Germany) were incubated with $100 \mu$ of patient serum diluted 1:101 for $1 \mathrm{~h}$ under shaking at $900 \mathrm{rpm}$. After five washing steps with the provided wash buffer, bound human IgG was detected with a provided conjugate. After another five washes, a color reaction was obtained using the provided substrate and stopped with stop solution. Absorbance was detected at $450 \mathrm{~nm}$ using a TECAN microplate reader (Tecan Life Science, Switzerland). Samples were measured in duplicates. Patients were considered positive for anti-SARS-CoV-2 antibodies if their signals were stronger than those of the provided cut-off control.

\section{Results}

\section{Clinical characteristics and laboratory data}

The patients' conditions were clinically classified into mild (P1-P4), moderate (P5-P10), and severe courses of the disease (P11 and P12) regarding their respiratory symptoms (Table 1). Additionally, we determined CRP, IL-6, and PCT serum levels to assess the inflammatory response of the patients. We used serum samples taken within the first 72 hours of hospital admission (day 1-3) and approximately one week later (day 6-10) (Table 1). All patients with a mild or moderate course of COVID-19 (P1-10), except for P3, had very high CRP (>100 mg/l) levels on admission, indicative of generalized high-grade inflammation. However, the CRP levels of these patients decreased after one week. In contrast, P11 and P12, who suffered from a severe course of COVID-19, had CRP levels below $100 \mathrm{mg} / \mathrm{l}$ on admission, and after one week, the CRP level of P12 increased to over $100 \mathrm{mg} / \mathrm{l}$, while the CRP level of P11 decreased. In contrast to CRP, PCT serum levels were not increased in most patients. Only P9 and P11 showed PCT serum levels of approximately $2 \mu \mathrm{g} / \mathrm{ml}$, suggesting an additional systemic bacterial infection. Upon admission to the hospital, the IL-6 levels of nine patients were below $150 \mathrm{ng} / \mathrm{l}$, indicative of local inflammation, as during pneumonia. Only P9 and P11 had IL-6 levels higher than $150 \mathrm{ng} / \mathrm{l}$, suggesting a systemic inflammatory response. However, one week later, the IL-6 levels of all patients decreased, except for P12, who showed an increase above $150 \mathrm{ng} / \mathrm{l}$. Interestingly, 11 out of 12 patients showed elevated CRP serum levels, but only one out of these 11 patients (P8) showed leucocyte counts above the reference range. Conversely, P3 had no elevated CRP level but an elevated leukocyte count. Nine of the 12 patients showed lymphocyte counts below the reference range. We also determined creatinine, transaminase (ASAT and ALAT), and lactate dehydrogenase (LDH) levels (Table 1). P9 and P11 had elevated creatinine levels associated with preexisting renal failure. Some patients showed elevated 
transaminases, but there was no evidence of acute liver failure. The calculated averages for LDH showed a rising level with the severity of the course of the disease, with $256 \mathrm{U} / \mathrm{l}$ at day 1 for the mild cohort, $573 \mathrm{U} / \mathrm{I}$ for the moderate cohort, and $473 \mathrm{U} / \mathrm{I}$ for the severe cohort. While the average levels of LDH declined after seven days in the mild and moderate cohorts to $229 \mathrm{U} / \mathrm{I}$ and $397 \mathrm{U} / \mathrm{I}$, respectively, the LDH level of the severe cohort increased to $622 \mathrm{U} / \mathrm{l}$.

\section{Recombinant SARS-CoV-2 proteins}

We used recombinant SARS-CoV-2 S, R, S1, and O proteins to detect anti-SARS-CoV-2 antibodies in the serum of COVID-19 patients by immunoblot analysis. First, recombinant SARS-CoV-2 proteins were analyzed by SDS-PAGE following Coomassie staining and by immunoblotting (Fig. 1a-b). The S protein with a calculated molecular mass of $138.8 \mathrm{kDa}$ showed two bands with apparent molecular masses of 175 and $35 \mathrm{kDa}$ in the Coomassie staining (Fig. 1a) and additional fragmentation bands in the immunoblot (Fig. 1b). To verify the presence of the fragmentation bands, we performed silver staining by SDS-PAGE (Fig. 1C). All immunoreactive bands ('S up' and 'S low') were considered when evaluating the immunoblots.

\section{Investigation of $\lg A,-M$, and $-G$ isotype antibodies by immunoblot analysis}

To investigate the IgA, -M, and -G-specific antibody response of COVID-19 patients, we analyzed the patient's serum by immunoblot. It should be noted that the immunoglobulin levels of the controls define a patient as positive or negative. Furthermore, $\lg \mathrm{A},-\mathrm{M}$, and $-\mathrm{G}$ antibodies with reactivity against SARS-CoV-2 proteins could also be detected in the controls (Supplementary Fig. S1-3). Most patients did not show antibodies directed against all four SARS-CoV-2 proteins. The S and S1 proteins were the most commonly detected proteins, while the R protein was the least commonly detected (Table 2). Additionally, the antibody reactivity (signal intensity) was stronger against the $S(S$ up) and $S 1$ proteins than against the R and 0 proteins (Supplementary Fig S1-3). The presence and signal intensity of antibodies directed against the 0 protein varied between the different isotype classes (Table 2 and Supplementary Fig. S1-3). However, IgM isotype antibodies directed against the 0 protein were detected in the majority of the patients. Only four out of 12 patients were positive for IgA isotype antibodies, as in most patients, fewer IgA antibodies were detected than in the controls. These patients were considered negative. Nevertheless, IgA antibodies with reactivity against SARS-CoV-2 proteins were present in the serum of all patients (Supplementary Fig. S2). Interestingly, IgA isotype antibodies in patients considered positive were reactive against the fragmented $S$ protein ( $\mathrm{S} \mathrm{low}$ ) but not the full-length $\mathrm{S}$ protein ( $\mathrm{Sup}$ ). This is also true for IgM isotype antibodies. In contrast, IgG isotype antibodies were frequently directed against non-fragmented $S$ protein (S up), while only four out of 12 patients showed antibodies directed against the fragmented $S$ protein (S low). All patient cohorts were positive for antibodies of almost all classes (Table 2). The only exception was IgA, which was negative in the patients in the severe group. To investigate the dynamics of 
the antibody response, we also analyzed the patient's serum one week after admission to the hospital. As the disease progressed, patients showed more anti-SARS-CoV-2 antibody reactivities; only for P5 IgM, P6 IgA, and P11 IgG did antibody reactivities decrease. However, P3 from the mild group was positive only for IgM antibodies on day 7, and P12 from the severe group was negative for all antibody classes at both time points. Interestingly, we found no correlation between the presence of antibodies and the future course of the disease.

\section{Enzyme-linked immunosorbent assay}

We analyzed the patients' serum with a commercially available ELISA kit kindly provided by Immundiagnostik AG (Germany) (Figure 2). The immunoassay detects human anti-SARS-CoV-2 S1 IgG isotype antibodies. The manufacturer provided positive and negative controls and a cut-off sample to facilitate the evaluation of the patients. By ELISA analysis, the serum of nine out of 12 patients was positive. Two of those were only positive on day 7 (P9 and P11), while the others were already positive on day 1 . There was a general increase in signal intensity observable in patients on day 7 . This increase was most prominent for P2, P8, P9, and P11. Conversely, P3 from the mild cohort, P6 from the moderate cohort, and P12 from the severe cohort showed no signals. The sera of the internal negative controls 1-3, which were used as cut-offs for the analysis of the immunoblots, were negative by ELISA.

\section{Comparison of immunoblot and ELISA analysis}

Both immunoblotting and ELISA use the same recombinant S1 protein purified in the same manner. Strikingly, no anti-SARS-CoV-2 antibodies could be detected by either method in the serum of P12, and only a few IgM antibodies could be detected by immunoblot at day 7 in the serum of P3. However, the comparison of the immunoblot and ELISA results showed discrepancies. P6 and P11 on day 1 were positive for IgG isotype antibodies directed against the $\mathrm{S} 1$ protein in immunoblot but not in ELISA analysis. $\mathrm{P} 2, \mathrm{P} 4, \mathrm{P} 5, \mathrm{P} 8$, and $\mathrm{P} 9$ show $\mathrm{IgG}$ antibodies directed against the $\mathrm{S} 1$ protein on day 1 and day 7 by ELISA but not by immunoblotting. However, these patients showed IgG antibodies directed against the $S$ protein in the immunoblot, indicating that anti-SARS-CoV-2 antibodies were present. ELISA (day 1: 7/12, day 7: 9/12; Table 3) showed more positive results for IgG isotype antibodies against the S1 protein than immunoblotting (day $1: 5 / 12$, day $7: 8 / 12$ ). By immunoblot analysis, most patients showed IgM antibodies directed against the $S$ protein (day $1: 7 / 12$, day $7: 9 / 12$ ). Interestingly, patients also frequently showed IgM antibodies directed against the 0 protein (day 1:6/12, day 7: 8/12). For IgG antibodies, only five out of 12 (day 1 ) and eight out of 12 (day 7) of the patients showed anti-S1 protein antibodies, while nine out of 12 (day 1 ) and ten out of 12 (day 7 ) showed anti-S protein antibodies (highest frequency). The R protein of SARS-CoV-2 was detected rather poorly in the immunoblot, indicating that there were only a few antibodies produced that are directed against this protein.

\section{Discussion}


The number of studies evaluating SARS-CoV-2 immunoassays is increasing steadily. However, immunoassays do not provide information about contaminations or cross-reactivities, which might lead to false high sensitivity and lower specificity. Therefore, we investigated the serum of 12 COVID-19 patients by immunoblot analysis for the presence of $\operatorname{lgM},-A$, and $-\mathrm{G}$ isotype antibodies directed against the SARS-CoV-2 S, R, S1, and O proteins.

The most striking result of the immunoblot analysis was the low number of patients who were positive for IgA antibodies directed against SARS-CoV-2 proteins. This may be the case since the serum is not the main localization of secreted, mature IgA antibodies. However, a recent study showed that $\lg \mathrm{G}$, IgM, and, to a lesser extent, IgA responses to spike and RBD in the serum positively correlated with matched saliva samples ${ }^{[12]}$. Nevertheless, our findings might also indicate an overall lack of IgA antibodies in immune defense. This could be a reason for SARS-CoV-2 reinfections ${ }^{[13,14]}$ due to a lack of IgA antibodies in the respiratory tract. However, the levels of detected IgA antibodies directed against SARS-CoV-2 proteins of the controls define a patient as positive or negative for IgA antibodies. The detection of anti-SARS-CoV-2 IgA antibodies in the controls is probably a result of cross-reactivity owing to previous infections with other coronaviruses or random antibodies. In most patients, fewer IgA antibodies than in the controls were detected; thus, these patients were considered negative. Nevertheless, IgA antibodies with reactivity against SARS-CoV-2 proteins were present in the patients' serum. To what extent these antibodies support immunity against the SARS-CoV-2 virus is unclear and can only be answered with functional, cell-based inhibition assays. This is true not only for $\lg A$ but also for $\lg M$ and $\lg G$ antibodies. Based on our results of the immunoblot analysis, we assume that even patients who underwent COVID-19 or vaccination may have a negative immunoassay result due to the threshold determined by the controls while being immune to the virus through existing antibodies.

A further, striking result of our study is that IgM and -A isotype antibodies predominantly recognize the degradation product of the S protein (S low). Perhaps this degradation product is presented by the MHC II complex, which leads to higher antibody production against it. Interestingly, the S and S1 proteins were more frequently detected than the R protein. An explanation could be that the RBD of SARS-CoV-2 spike protein $(R)$ is usually not accessible and masked by the rest of the protein ${ }^{[15]}$. Although antibodies against the RBD may be effective in inhibiting the virus from penetrating cells and may be suitable as a vaccine antigen ${ }^{[16]}$, the RBD alone is unsuitable as an antigen for diagnostic purposes. In contrast, the ORF8 protein is well detected through $\lg M$ antibodies but not by $\lg A$ or $\lg G$ antibodies. The function of ORF8 is still under investigation ${ }^{[17]}$. However, there are reports that it is involved in suppressing the host's immune response through the downregulation of $\mathrm{MHC} \mathrm{I} \mathrm{complexes}{ }^{[18]}$ and suppressing interferon type I [19]. Thus, ORF8 is a promising pharmaceutical target but is not suitable for the detection of $\lg A$ and $-G$ antibodies.

We found differences between the results of the immunoblot and ELISA analysis of IgG isotype antibodies directed against the $\mathrm{S} 1$ protein. Sometimes, antibodies were detected by immunoblot but not by ELISA, and vice versa. The virus proteins are denatured for the immunoblot, while the ELISA utilizes 
their native form, therefore, detecting three-dimensional epitopes rather than linear ones as in the immunoblot which might lead to differing results. According to the immunoblot analysis, the $\mathrm{S} 1$ protein is not necessarily the best antigen for detecting IgM isotype antibodies, as more patients were positive for antibodies directed against the $S$ protein. Using a combination of the $S$ protein and the $O$ protein could prove to be the best for the detection of IgM isotype antibodies, as the 0 protein is a potent antigen for detecting COVID-19 patients in early to late stages of infection ${ }^{[20]}$. For the detection of IgG isotype antibodies, our data suggest that the use of the $S$ protein should be sufficient to identify positive patients. However, there is evidence that the use of the S1 domain instead of the full-length S protein leads to less cross-reactivity ${ }^{[21]}$.

Based on our results, we wondered if the presence of antibodies could help to stratify the patients, but we could not observe any correlation between the presence of anti-SARS-CoV-2 antibodies and the course of the disease. We found high levels of the inflammatory parameters CRP and IL- 6 but not of leukocyte count and PCT and a rising LDH level with the severity of the course of disease. In the context of a COVID-19 disease, an excessive inflammatory response also called a hyperinflammatory syndrome can occur. Patients with hyperinflammatory syndrome may benefit from therapy targeting cytokine storm syndrome. Recently, a set of clinical criteria for COVID-19-associated hyperinflammatory syndrome was proposed ${ }^{[22]}$. Furthermore, we observed a correlation between CRP and IL- 6 as well as lymphocyte count and antibody reactivity. Within one week, CRP and IL- 6 levels decreased, while lymphocyte counts and antibody reactivity increased in patients $1-11$. Conversely, for P12, who was not immunosuppressed and showed no antibody reactivity, the lymphocyte count decreased, while CRP and IL- 6 levels increased. This highlights the importance of antibodies in the defense against SARS-CoV-2 infections. However, we cannot provide any explanation for why the patients in the severe cohort showed lower values for CRP and IL- 6 than those in the moderate and mild cohorts. Perhaps the lower values are a result of a compensatory anti-inflammatory response.

In conclusion, we found evidence for antibody cross-reactivity, which questions a reliable assessment of serum samples with a negative result for anti-SARS-CoV-2 antibodies tested by immunoassay.

Nevertheless, for the detection of anti-SARS-CoV-2 IgG antibodies, our data suggest that the use of the $S$ protein in immunoassays should be sufficient to identify positive patients. Using a combination of the $S$ protein and the $O$ protein could prove to be the best for the detection of patients positive for anti-SARS-CoV-2 IgM antibodies. In this study, the antibody response alone was not decisive for the course of the disease. To assess the severity of a SARS-CoV-2 infection, attention should be paid to the parameters CRP, IL-6, and LDH.

\section{References}

1 Perlman, S. \& Netland, J. Coronaviruses post-SARS: update on replication and pathogenesis. Nat Rev Microbio/ 7, 439-450, doi:10.1038/nrmicro2147 (2009). 
2 Huang, C. et al. Clinical features of patients infected with 2019 novel coronavirus in Wuhan, China. Lancet 395, 497-506, doi:10.1016/S0140-6736(20)30183-5 (2020).

3 Ksiazek, T. G. et al. A novel coronavirus associated with severe acute respiratory syndrome. N Engl J Med 348, 1953-1966, doi:10.1056/NEJMoa030781 (2003).

4 Zaki, A. M., van Boheemen, S., Bestebroer, T. M., Osterhaus, A. D. \& Fouchier, R. A. Isolation of a novel coronavirus from a man with pneumonia in Saudi Arabia. N Engl J Med 367, 1814-1820, doi:10.1056/NEJMoa1211721 (2012).

5 Domling, A. \& Gao, L. Chemistry and Biology of SARS-CoV-2. Chem 6, 1283-1295, doi:10.1016/j.chempr.2020.04.023 (2020).

6 Tan, Y., Schneider, T., Leong, M., Aravind, L. \& Zhang, D. Novel Immunoglobulin Domain Proteins Provide Insights into Evolution and Pathogenesis Mechanisms of SARS-Related Coronaviruses. bioRxiv, 2020.2003.2004.977736, doi:10.1101/2020.03.04.977736 (2020).

7 Cheng, M. P. et al. Diagnostic Testing for Severe Acute Respiratory Syndrome-Related Coronavirus 2: A Narrative Review. Ann Intern Med 172, 726-734, doi:10.7326/M20-1301 (2020).

8 Zhao, J. et al. Development and evaluation of an enzyme-linked immunosorbent assay for detection of antibodies against the spike protein of SARS-coronavirus. J Clin Viro/ 33, 12-18, doi:10.1016/j.jcv.2004.09.024 (2005).

9 Zhao, J. et al. A study on antigenicity and receptor-binding ability of fragment 450-650 of the spike protein of SARS coronavirus. Virology 359, 362-370, doi:10.1016/j.virol.2006.09.022 (2007).

10 Organization, W. H. Clinical management of severe acute respiratory infection (SARI) when COVID19 disease is suspected. Interim guidance. Pediatria i Medycyna Rodzinna 16, 9-26, doi:10.15557/PiMR.2020.0003 (2020).

11 Kowarz, E., Loscher, D. \& Marschalek, R. Optimized Sleeping Beauty transposons rapidly generate stable transgenic cell lines. Biotechnol J 10, 647-653, doi:10.1002/biot.201400821 (2015).

12 Isho, B. et al. Persistence of serum and saliva antibody responses to SARS-CoV-2 spike antigens in COVID-19 patients. Sci Immuno/ 5, doi:10.1126/sciimmunol.abe5511 (2020).

13 Tillett, R. L. et al. Genomic evidence for reinfection with SARS-CoV-2: a case study. Lancet Infect Dis, doi:10.1016/S1473-3099(20)30764-7 (2020).

14 To, K. K.-W. et al. COVID-19 re-infection by a phylogenetically distinct SARS-coronavirus-2 strain confirmed by whole genome sequencing. Clinical Infectious Diseases, doi:10.1093/cid/ciaa1275 (2020). 
15 Walls, A. C. et al. Structure, Function, and Antigenicity of the SARS-CoV-2 Spike Glycoprotein. Cell 181, 281-292 e286, doi:10.1016/j.cell.2020.02.058 (2020).

$16 \mathrm{Ju}, \mathrm{B}$. et al. Human neutralizing antibodies elicited by SARS-CoV-2 infection. Nature 584, 115-119, doi:10.1038/s41586-020-2380-z (2020).

17 Flower, T. G. et al. Structure of SARS-CoV-2 ORF8, a rapidly evolving coronavirus protein implicated in immune evasion. bioRxiv, doi:10.1101/2020.08.27.270637 (2020).

18 Zhang, Y. et al. The ORF8 Protein of SARS-CoV-2 Mediates Immune Evasion through Potently Downregulating MHC-I. bioRxiv, 2020.2005.2024.111823, doi:10.1101/2020.05.24.111823 (2020).

$19 \mathrm{Li}$, J. Y. et al. The ORF6, ORF8 and nucleocapsid proteins of SARS-CoV-2 inhibit type I interferon signaling pathway. Virus Res 286, 198074, doi:10.1016/j.virusres.2020.198074 (2020).

20 Hachim, A. et al. ORF8 and ORF3b antibodies are accurate serological markers of early and late SARS-CoV-2 infection. Nat Immunol 21, 1293-+, doi:10.1038/s41590-020-0773-7 (2020).

21 Mueller, L. et al. Sensitivity of commercial Anti-SARS-CoV-2 serological assays in a highprevalence setting. medRxiv, 2020.2006.2011.20128686, doi:10.1101/2020.06.11.20128686 (2020).

22 Webb, B. J. et al. Clinical criteria for COVID-19-associated hyperinflammatory syndrome: a cohort study. Lancet Rheumatol, doi:10.1016/S2665-9913(20)30343-X (2020).

\section{Declarations}

\section{Data availability}

All data generated or analysed during this study are included in this published article (and its Supplementary Information files).

\section{Acknowledgments}

We owe special thank you to Johannes Ruthard and Dr. Dzemal Elezagic from the Institute for Clinical Chemistry of the Medical Faculty of the University of Cologne for fruitful discussions and help with the acquisition of samples and the patients' clinical data. From the Institute for Biochemistry II of the Medical Faculty of the University of Cologne, we thank Prof. Dr. Mats Paulsson for carefully reading the manuscript and Semra Oezcelik for her excellent technical help.

\section{Additional information}

\section{Author contributions}

Conceptualization: DM, MK, and AK; Methodology: DM, MK, GH, and AK; Sample collection: DM, GH, JS, OC, and TS; Formal analysis: DM, GH and AK; Investigation: DM, MK and AR; Writing-first draft 
preparation: DM and AK; Writing-review, and editing: JS, OC, MK, AR, TS, and AK.

\section{Competing interests}

There are no competing financial and/or non-financial interests in relation to the work described. The authors declare no competing interests.

\section{Tables}

Table 1: Clinical parameters of the patients.

\begin{tabular}{|c|c|c|c|c|c|c|c|c|c|c|c|c|c|}
\hline \multicolumn{2}{|c|}{ Patient Nr. } & $\begin{array}{l}\text { Sex } \\
\text { Age }\end{array}$ & $\begin{array}{l}\text { Days since } \\
\text { admission }\end{array}$ & $\begin{array}{c}\text { CRP } \\
{[\mathrm{mg} / \mathrm{l}]}\end{array}$ & $\begin{array}{c}\mathrm{PCT} \\
{[\mu \mathrm{g} / 1]}\end{array}$ & $\begin{array}{c}\mathrm{IL}-6 \\
{[\mathrm{ng} / \mathrm{l}]}\end{array}$ & $\begin{array}{c}\text { Leuco- } \\
\text { cytes } \\
\text { [1E9/1] }\end{array}$ & $\begin{array}{c}\text { Lympho- } \\
\text { cytes } \\
\text { [1E9/1] }\end{array}$ & $\begin{array}{l}\text { Cerati- } \\
\text { nine } \\
{[\mathrm{mg} / \mathrm{dl}]}\end{array}$ & $\begin{array}{l}\text { ASAT } \\
{[\mathrm{U} / 1]}\end{array}$ & $\begin{array}{l}\text { ALAT } \\
{[\mathrm{U} / 1]}\end{array}$ & $\begin{array}{c}\mathrm{LDH} \\
{[\mathrm{U} / 1]}\end{array}$ & $\begin{array}{c}\text { LDH } \\
\text { aver- } \\
\text { age }\end{array}$ \\
\hline \multirow{3}{*}{$\begin{array}{l}\text { Mild } \\
\text { CoD }\end{array}$} & \multirow[t]{2}{*}{1} & W & 1 & 282.3 & 0.2 & 89 & 1.7 & 0.36 & 0.46 & 18 & 12 & 188 & \multirow{4}{*}{$\begin{array}{c}\text { Day } 1 \\
256\end{array}$} \\
\hline & & 31 & 7 & 104.4 & 0.1 & 21 & 3.6 & 0.37 & 0.45 & 33 & 29 & 182 & \\
\hline & \multirow[t]{2}{*}{2} & $\mathrm{~W}$ & 2 & 217.3 & 0.1 & 43 & 8.9 & 0.37 & 0.91 & 48 & 42 & 248 & \\
\hline \multirow{5}{*}{$\begin{array}{c}\text { WHO } \\
3\end{array}$} & & 62 & 7 & 41.9 & 0.1 & 9 & 7.6 & 1.01 & 0.83 & 53 & 61 & 232 & \\
\hline & \multirow[t]{2}{*}{3} & $\mathrm{~W}$ & 3 & 6.1 & 0.0 & 2 & 13.5 & 0.86 & 0.62 & 15 & 23 & 230 & \multirow{4}{*}{$\begin{array}{c}\text { Day } 7 \\
229\end{array}$} \\
\hline & & 89 & 7 & 1.0 & 0.0 & 3 & 16.3 & 1.40 & 0.59 & 18 & 25 & 268 & \\
\hline & \multirow[t]{2}{*}{4} & $\mathrm{M}$ & 3 & 115.7 & 0.1 & 38 & 8.5 & 1.54 & 0.87 & 48 & 100 & 361 & \\
\hline & & 56 & 9 & 5.5 & 0.1 & 4 & 8.6 & 2.20 & 0.81 & 40 & 125 & 234 & \\
\hline \multirow{12}{*}{$\begin{array}{l}\text { Mod- } \\
\text { erate } \\
\text { CoD }\end{array}$} & \multirow[t]{2}{*}{5} & $\mathrm{~W}$ & 1 & 110.6 & 0.1 & n.a. & 7.7 & 0.90 & 0.77 & 166 & 64 & 757 & \multirow{6}{*}{$\begin{array}{c}\text { Day } 1 \\
573\end{array}$} \\
\hline & & 50 & 7 & 25.8 & 0.1 & n.a. & 9.0 & 1.72 & 0.57 & 49 & 62 & 445 & \\
\hline & \multirow[t]{2}{*}{6} & $\mathrm{M}$ & 1 & 113.8 & 1.5 & 40 & 3.8 & 0.51 & 1.26 & 165 & 81 & 797 & \\
\hline & & 53 & 6 & 89.3 & 0.3 & 114 & 5.6 & 0.92 & 1.21 & 114 & 143 & 585 & \\
\hline & \multirow[t]{2}{*}{7} & $\mathrm{M}$ & 1 & 124.9 & 0.1 & 127 & 11.3 & 1.15 & 0.88 & 28 & 62 & 331 & \\
\hline & & 58 & 6 & 87.0 & 0.2 & 11 & 5.1 & 1.22 & 0.71 & 43 & 63 & 239 & \\
\hline & \multirow[t]{2}{*}{8} & M & 2 & 124.6 & 0.4 & 94 & 24.1 & 12.59 & 1.08 & 124 & 65 & 549 & \multirow{6}{*}{$\begin{array}{c}\text { Day } 7 \\
397\end{array}$} \\
\hline & & 50 & 9 & 6.4 & 0.1 & 4 & 14.8 & 10.72 & 0.83 & 71 & 144 & 378 & \\
\hline & \multirow[t]{2}{*}{9} & $\mathrm{M}$ & 2 & 226.4 & 4.3 & 571 & 5.2 & 0.29 & 8.01 & 68 & 24 & 536 & \\
\hline & & 74 & 9 & 52.1 & 1.0 & 31 & 6.0 & 1.26 & 6.19 & 21 & 15 & 322 & \\
\hline & \multirow[t]{2}{*}{10} & $\mathrm{~W}$ & 2 & 111.6 & 1.4 & 48 & 10.0 & 1.51 & 1.91 & 164 & 42 & 470 & \\
\hline & & 69 & 10 & 1.9 & 0.0 & 67 & 13.4 & 3.20 & 0.52 & 45 & 54 & 415 & \\
\hline \multirow{4}{*}{$\begin{array}{c}\text { Severe } \\
\text { CoD }\end{array}$} & \multirow[t]{2}{*}{11} & $\mathrm{~W}$ & 3 & 86.5 & 0.5 & 191 & 3.9 & 0.51 & 2.58 & 28 & 13 & 227 & \multirow{2}{*}{$\begin{array}{c}\text { Day } 1 \\
473\end{array}$} \\
\hline & & 88 & 9 & 35.9 & 1.9 & 47 & 3.9 & 0.60 & 1.43 & 33 & 10 & 324 & \\
\hline & \multirow[t]{2}{*}{12} & $\mathrm{M}$ & 3 & 53.0 & 0.1 & 33 & 5.1 & 1.00 & 0.91 & 43 & 65 & 719 & \multirow{2}{*}{$\begin{array}{c}\text { Day } 7 \\
622\end{array}$} \\
\hline & & 65 & 7 & 200.4 & 0.3 & 154 & 3.9 & 0.75 & 0.95 & 88 & 65 & 920 & \\
\hline \multicolumn{2}{|c|}{$\begin{array}{c}\text { Reference } \\
\text { values }\end{array}$} & $\begin{array}{l}\mathrm{W} \\
\mathrm{M}\end{array}$ & & $<5.0$ & $<0.1$ & $<8$ & $4.4-11.3$ & $1.26-3.35$ & $\begin{array}{l}0.50-0.90 \\
0.50-1.10\end{array}$ & $\begin{array}{l}<35 \\
<50\end{array}$ & $\begin{array}{l}<35 \\
<50\end{array}$ & \multicolumn{2}{|c|}{$<250$} \\
\hline
\end{tabular}

$\mathrm{CoD}=$ course of the disease. The age of the patients is reported in years

Table 2: Evaluation of the patient immunoblots. 


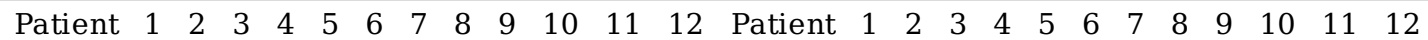
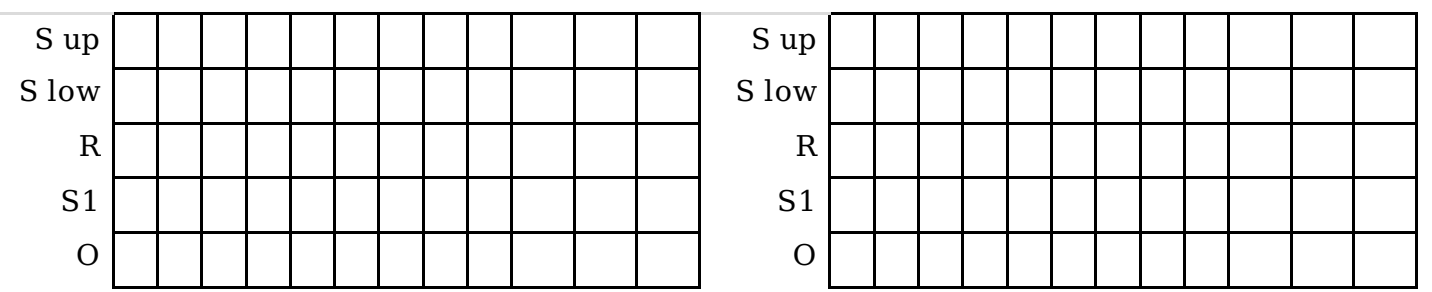

IgA

Day 1

Day 7

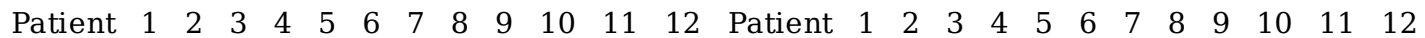

S up
S low

S up
S low

IgG

Day 1

Day 7

$\begin{array}{llllllllllllllllllllllllll}\text { Patient } & 1 & 2 & 3 & 4 & 5 & 6 & 7 & 8 & 9 & 10 & 11 & 12 & \text { Patient } & 1 & 2 & 3 & 4 & 5 & 6 & 7 & 8 & 9 & 10 & 11 & 12\end{array}$

S up
S low

S up
S low \begin{tabular}{rl|l|l|l|l|l|l|l|l|l|l|}
\hline \\
\cline { 2 - 10 } \\
\cline { 2 - 11 } \\
$\mathrm{S}$
\end{tabular}

The serum of COVID-19 patients 1-12, either taken on the day of admission to the hospital (day 1) or one week later (day 7), was used as a primary antibody to detect the recombinant SARS-CoV-2 proteins S, R, S1, and O by immunoblotting. The presence of anti-SARS-CoV-2 antibodies was determined for IgM, -A, and -G isotype antibodies. A patient was labeled positive for antibodies (filled boxes) if the absolute band intensity of the patient exceeded the highest band intensity of the controls. All immunoblots are depicted in Supplementary Fig. S1-3.

Table 3: Comparison of the results from the immunoblot and ELISA analysis.

\begin{tabular}{|c|c|c|c|c|c|c|c|c|c|c|c|c|c|}
\hline & \multicolumn{12}{|c|}{ Immunoblot } & \multirow{2}{*}{\begin{tabular}{|c|} 
ELISA \\
$\operatorname{Ig} G$ \\
\end{tabular}} \\
\hline & \multicolumn{4}{|c|}{$\operatorname{Ig} M$} & \multicolumn{4}{|c|}{$\operatorname{Ig} \mathrm{A}$} & \multicolumn{4}{|c|}{ IgG } & \\
\hline & $\mathrm{S}$ & $\mathrm{R}$ & S1 & $\mathrm{O}$ & $\mathrm{S}$ & $\mathrm{R}$ & S1 & O & $\mathrm{S}$ & $\mathrm{R}$ & S1 & 0 & $\mathrm{~S} 1$ \\
\hline $\begin{array}{c}\text { Positive Results } \\
\text { Day } 1(n=12)\end{array}$ & 7 & 2 & 3 & 6 & 1 & 2 & 1 & 1 & 9 & 1 & 5 & 3 & 7 \\
\hline $\begin{array}{c}\text { Positive Results } \\
\text { Day } 7(n=12)\end{array}$ & 9 & 2 & 4 & 8 & 1 & 2 & 3 & 2 & 10 & 1 & 8 & 4 & 9 \\
\hline
\end{tabular}

Positive results for each category were counted. $n=12$ 


\section{Figures}

a

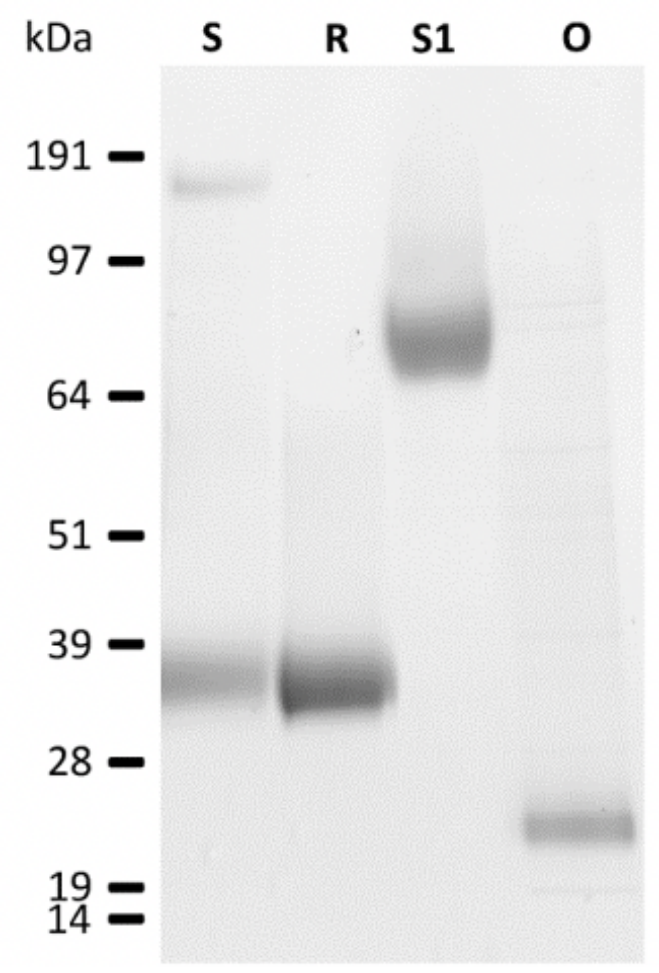

b
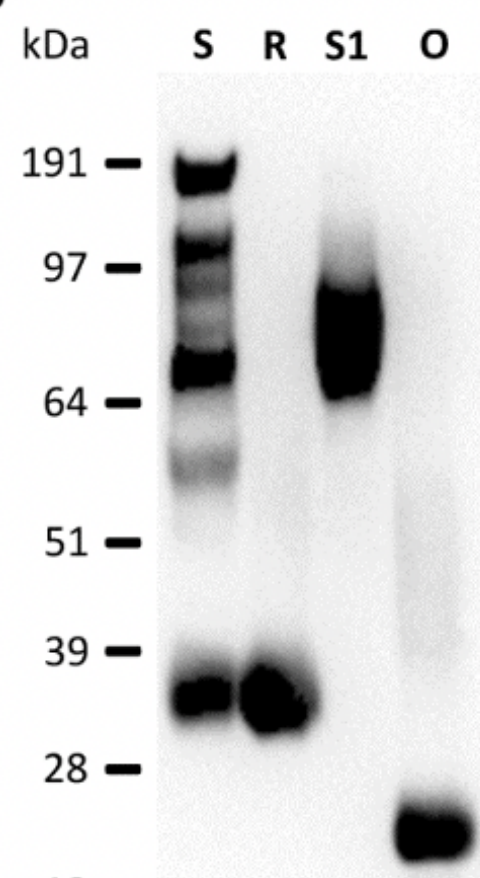

${ }_{14}^{19}=$
C $\mathrm{kDa} \quad \mathrm{S}$

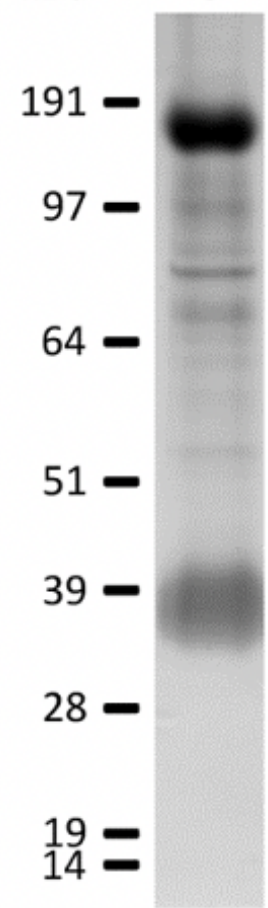

\section{Figure 1}

Recombinant SARS CoV 2 proteins. (a) $412 \%$ Bis Tris gel loaded with $4 \mu \mathrm{g}$ of SARS CoV 2 spike protein $(\mathrm{S})$, the RBD of spike protein (R), subunit 1 of the spike protein (S1), and ORF8 protein (0) stained with Coomassie brilliant blue R 250. (b) Immunoblot with $2 \mu \mathrm{g}$ of Strep tagged SARS CoV 2 proteins loaded in the same manner as the Coomassie gel in a detected with a Strep Tactin HRP conjugate. (c) Silver staining with $2 \mu \mathrm{g}$ of SARS CoV 2 proteins loaded in the same manner as the Coo-massie gel in a. 


\section{Anti-SARS-CoV-2 S1 protein IgG ELISA}

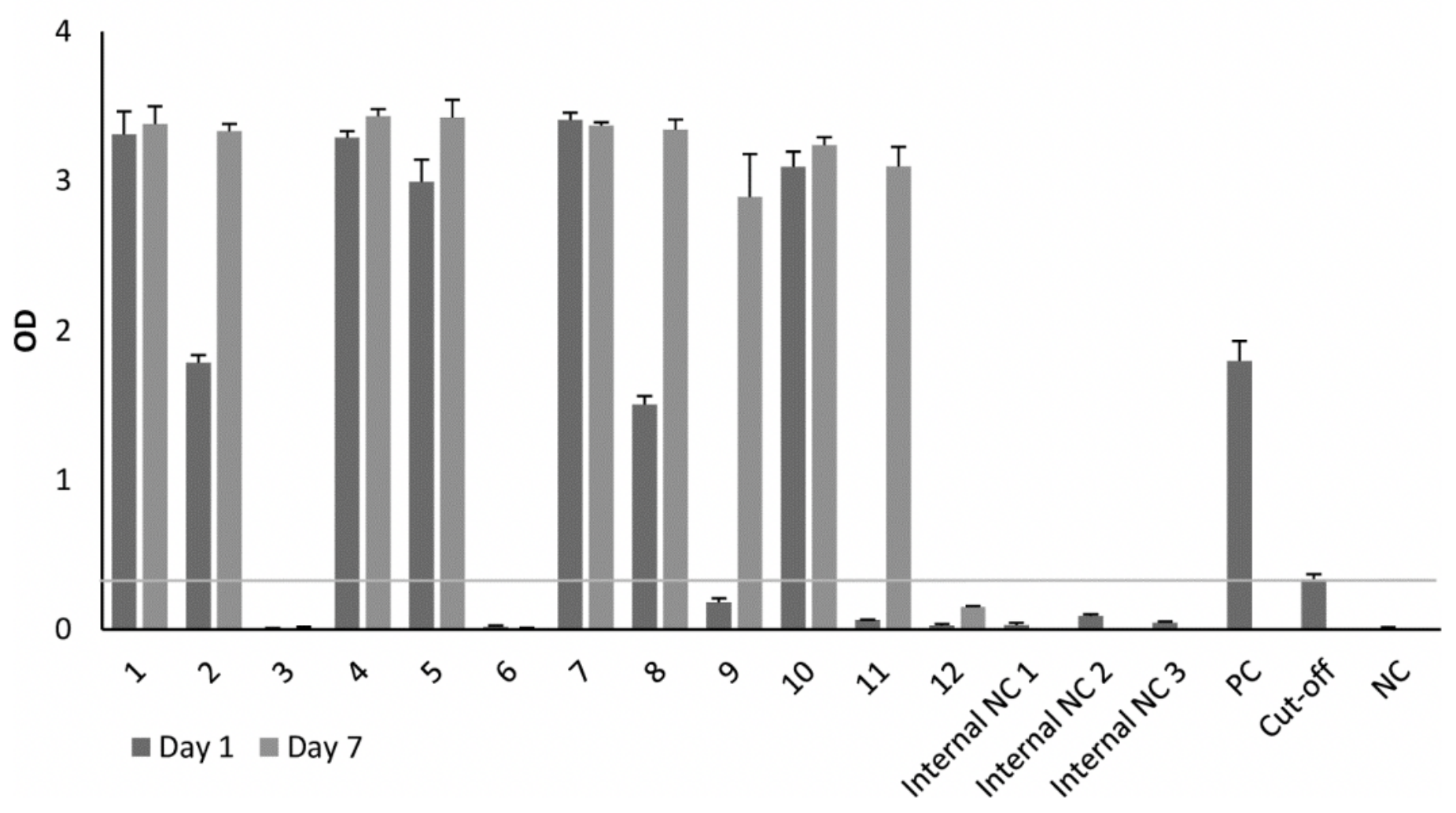

Figure 2

Anti SARS CoV 2 S1 protein IgG ELISA. Patient serum was tested for IgG isotype antibodies directed against subunit 1 of the SARS CoV 2 spike protein (S1). Patients with a higher optical density (OD) measured at $\lambda=450 \mathrm{~nm}$ than the provided cut off were labeled positive for IgG isotype antibodies directed against the $\mathrm{S} 1$ protein. Average ODs and standard deviations are shown in the bar chart. $\mathrm{n}=3$.

\section{Supplementary Files}

This is a list of supplementary files associated with this preprint. Click to download.

- 20201113SupplementaryInformationScientificReportseddited.docx 